Mathematical Modelling and Analysis

Volume 16 Number 2, June 2011, 173-198

Doi:10.3846/13926292.2011.578264

(C) Vilnius Gediminas Technical University, 2011
www.tandf.co.uk/journals/TMMA

Publisher: Taylor\&Francis and VGTU

Online ISSN: 1648-3510

Print ISSN: 1392-6292

\title{
Mathematics in the Context of Fuzzy Sets: Basic Ideas, Concepts, and Some Remarks on the History and Recent Trends of Development
}

\author{
Alexander Šostaks ${ }^{a, b}$ \\ ${ }^{a}$ Department of Mathematics, University of Latvia \\ Zellu iela 8, Riga, LV-1002 \\ ${ }^{b}$ Institute of Mathematics and Computer Science, University of Latvia \\ E-mail(corresp.): sostaks@latnet.lv
}

Received November 15, 2010; revised March 10, 2011; published online May 1, 2011

\begin{abstract}
The main aim of this paper is to discuss the basic ideas and concepts of the so called "Fuzzy Mathematics" and to give a brief survey of the history and of some trends in recent development of mathematics and its applications in the context of fuzzy sets. As a potential reader we imagine a mathematician, who is not working in the field of "fuzzy mathematics", but wishes to have some idea about this vast field in modern science.
\end{abstract}

Keywords: fuzzy set, fuzzy logic, fuzzy real number.

AMS Subject Classification: 03B52; 03E72; 08A72; 28E10; 34A07; 54A40.

\section{Introduction and Motivation}

Since the inception of the concept of an abstract set by Georg Cantor at the end of the nineteenth century, the sets have firmly occupied one of the central places in mathematics, and they make the foundation for many branches of theoretical as well as of applied mathematics. However, being a perfect mathematical concept, in practice people usually have to deal not with usual, or crisp, sets, that is with the sets as they are understood in mathematics, but with setlike conglomerates having vague, non-sharp or imprecise borders. Just a few examples to illustrate this assertion.

Consider the set of all citizens of some state, say Lithuania. It is a real set, a subset with sharp borders of the set of all people in the world. Every person either belongs to this set or not. On the other hand consider "the set" of young Lithuanian people. Obviously, this is not a set in the precise mathematical sense: there is no natural age border after which a man or a woman stops to be young and becomes non-young. Moreover, if for some purposes, e.g. in case a competition of applicants for research grants among 
young scientists is announced and according to regulations a person is called young if he/she is younger than 30 years, this division into young and non-young people is artificial and even may cause problems for a committee awarding such grants. Say if today is the applicant's $30^{t h}$ birthday and just today he has submitted the project - whether the committee, working formally, consider this application as valid? Anyway the set of young people is a not a set but a "set-like conglomerate", or "a fuzzy set" and this conglomerate has non-sharp, vague, imprecise borders.

Another example. In mathematics and its applications one has often to deal with such sets as, say, the set of all real numbers larger than 10. Obviously it is an example of a typical usual set. On the other hand quite often we have to deal with such set-like conglomerates as "the set" of all real numbers which are approximately equal to 10, or "the set" of all numbers which are much larger than 10. Clearly, the borders of such conglomerates are not sharp and they make examples of fuzzy sets.

Since ancient times most reasonings in mathematics are grounded on bivalent, or binary, logic. According to this logic every statement in mathematics should be either true or false. However, in practice dealing with different problems one often encounters statements which are more or less true, or true to a large extent, or are unlikely, etc. Such statements cannot be classified and studied in the frames of the classical, or bivalent, logic, but just belong to the subject of what is called fuzzy logic. Again we present a couple of examples.

Consider the statements "If $a$ and $b$ are real negative numbers, then the product $a \cdot b$ is positive" and "If $a$ is larger than $b$ and $n \geq 10, a, b, n$ being natural numbers, then $a^{n}$ is much larger than $b^{n}$ ". Obviously, the first one is a precise mathematical statement which is true, while the second statement could be viewed as a fuzzy statement which, is more or less true.

Another example. Consider two statements "If it is raining there are clouds in the sky" and "If there are clouds in the sky, then possibly it will rain". The first one is formulated according to the laws of bivalent logic, while the second one can be viewed as a statement in fuzzy logic.

Since the introduction of the concept of a fuzzy set and laying down the foundations of fuzzy logic in 1965 by L.A. Zadeh [94], the interests of many researchers in theoretical mathematics as well as in applications of mathematics in other sciences were directed to applying fuzzy sets and rules of fuzzy logics in their work. Many research papers on this subject were published, several regular conferences devoted to the problems of "Fuzzy Mathematics" were organized. For example, every 4 years the International Congress of Fuzzy Association (IFSA) takes place. Since 1979 in Linz, Austria an annual "Linz Seminar on Fuzzy Sets" is organized, every time its programme is devoted to a particular topic of research. Since 1992 in Slovakia a biannual conference "Fuzzy Sets: Theory and Applications (FSTA)" is held. At present there are several journals specializing in publishing works in "Fuzzy Mathematics" and its applications, in particular International Journal of Fuzzy Sets and Systems (launched in 1978 and included in the ISI list) and Iranian Journal of Fuzzy Systems (launched in 2004 and included in the extended ISI list). Also other journals which have a sufficiently broad spectrum of acceptable research pa- 
pers, and the Journal of Mathematical Modelling and Analysis among them, publishes manuscripts where mathematical structures on the basis of fuzzy sets are studied or applied. This was one of the reasons why the organizing committee of the annual $15^{\text {th }}$ conference Mathematical Modelling and Analysis, which was held in Druskininkai, Lithuania in May 26-30, 2010, has invited the author of this paper to give a key-note talk. In this talk, the basic ideas and concepts of the theory of fuzzy sets were presented and examples illustrating the interplay of fuzzy sets in the branches of classical mathematics were given.

The present work is an essentially extended version of the talk given at MMA2010 conference, its main aim is to give a very brief introduction into what we call "Mathematics in the context of fuzzy sets" for mathematicians who are not professionals in this field but who want to have some idea of this area of modern mathematics.

\section{History}

\subsection{Fuzzy sets and fuzzy reasonings: prehistory 1900-1965}

The comprehension that in real situations discourses do not always lead either to true or to false statements and that there are many statements between true and false, that is statements of gradual truth was, obviously, not a new idea in science. As well as not a new was the idea, that not all objects of the real world in respect of some property can be classified as "white" or "black". There can be many objects of different "shadows of grey colors", that is objects having a given property within a certain degree. In particular, such idea was discussed already in Aristotle's works, see e.g. [4]. However, in the modern times scientists started to express serious interest in the problem of gradual truth and the related problem of having a property within a certain degree only since the end of the $19^{\text {th }}$ century. It is also worth to note, that first people to be mentioned in this respect are scientists of broad scientific interests, scientists known both as philosophers and researchers in exact sciences. Charles Peirce (1839-1914 USA), known as chemists, philosopher and mathematician, wrote "Logicians have too much neglected the study of vagueness, not suspecting the important part it plays in mathematical thought" [60]. Bertrand Arthur William Russel (1872-1970 Great Britain), an outstanding mathematician, logician, writer and philosopher, discussed these problems in his treatise "An introduction to Mathematical Philosophy" [70]. In 1937 there was published the monograph "Vagueness: an exercise in logical analysis" by Max Black (1909 Azerbaijan 1970 USA), a philosopher and a researcher in the field of quantum mechanics [10]. In this monograph the author considered "consistency profiles" in order to characterize quantities without clear borders which he called "vague symbols" and discussed the importance of vague symbols for philosophical problems. Black's vague symbols are closely related to interval-type fuzzy numbers described in this paper in Section 4.1.

Probably the first scientist who tried to study the problem of uncertainty from the point of view of mathematical logic was Jan Łukasiewicz (Poland 1878 - Ireland 1956). He developed a new logical system, in which statements can 
be not only false or true, but can be also true/false to a certain degree. In his lecture on March 7, 1918 at the Warsaw University he announced that his logical system "is as coherent and self-contained as Aristotle's logic but which is much richer in laws and formulae." This logical system is actually a threevalued case of what is understood by Łukasiewicz logic now, see Section 5.2, see also Łukasiewicz's paper [47]. Independently of Łukasiewicz and starting from different premises, another system of many-valued logics was discovered by Emil Post (Russia 1897 - USA 1954) [61].

One more mathematician whose contribution to the "prehistory" of Fuzzy Mathematics, should be mentioned, was Karl Menger (Austria 1902 - USA 1985). K. Menger suggested to develop a theory in which the relation $\in$ "element belongs to a set" (which is in the basis of the Cantor set theory) is replaced by the probability of an element belonging to a set. In his paper Probabilistic geometry, [49] he used objects which can be considered as precursors of fuzzy points (see Section 3.3) and which he called hazy sets.

\subsection{Fuzzy sets and fuzzy logics: early history 1965-1975}

As we tried to show in the previous section, the ideas to find a mathematical concept appropriate to describe objects which are not precisely defined as well as to deal with statements for the validity of which one cannot give a monosemantic answer "yes" or "no", has emerged in the works of many scientists during the first half of the $20^{\text {th }}$ century. However the credits of founding the theory of fuzzy sets (and "inventing" the term "fuzzy set" itself) are given to the professor of the Berkley University L.A. Zadeh. L.A. Zadeh was born in 1921, in Baku, at that time the capital of the Soviet Azerbaijan. Later he moved with his parents to Iran (they were the Iranian citizens) where they lived till 1944. In 1944 Zadeh went for studies to the USA. In 1951 he has received doctor degree in electrical engineering, and in 1963 was appointed as the head of the department of electrical engineering at the University of Berkeley. In 1956 he met S. Kleene (1909-1994), an outstanding logician, the author of the famous "Introduction to metamathematics" [34]. Friendship with Kleene had a great influence on Zadeh. In particular, this influence showed itself in his idea to use many-valued logics in order to describe the behaviour of complex electrical systems. Later this idea has developed into the concept of a fuzzy set. The foundations of the theory of fuzzy sets were first developed [94]. This work was followed by many subsequent Zadeh's papers in which different aspects of the theory of fuzzy sets and fuzzy logic and related problems were considered. ${ }^{1}$

The inception of fuzzy sets by Zadeh did not remain unnoticed: in the next decade there were published several important papers considering fuzzy sets in theoretical mathematics (Topology: C.L. Chang [12], B. Hutton [29], J.A. Goguen [23]; Algebra: A. Rosenfeld [69], D.N. Mordenson and D.S. Malik [51]; Measure and integral theory: L.A. Zadeh [95], M. Sugeno [86]) and in applied sciences (Decision making: R. Bellman and L. Zadeh [8], System analysis:

\footnotetext{
${ }^{1}$ A reader interested in the biography of this outstanding scientist and person can refer to the book written by Zadeh's wife [93] or to the book of Zadeh's student and a popularizer of the ideas of fuzzy sets and fuzzy logic B. Kosko [39].
} 
C.R. Negoita and D.A. Ralescu [53], etc.) A notable contribution to the field of "Fuzzy Mathematics" was done by J.A. Goguen [22], who introduced an important and natural generalization of a fuzzy set, the so called an L-fuzzy set (where $L$ could be any complete lattice or even a cl-monoid, [9]) thus essentially enlarging the scope of possible applications of the original concept of a fuzzy set and enriching the mathematical theories in the context of fuzzy sets. In this period also some works criticizing the whole concept of a fuzzy set were published, see e.g. [3]. We refer to [14] for the discussion around this criticism.

\subsection{Fuzzy sets and fuzzy logics: development after 1975}

In the last quarter of the $20^{t h}$ century and at the beginning of the $21^{\text {th }}$ century the number of works where fuzzy sets have been used increased in an avalanche way. There are thousands of works in traditional branches of theoretical mathematics in the context of fuzzy sets: Topology, Algebra, Measure and Integral Theory, Differential Equations, Probability theory and Mathematical Statistics, Mathematical Modeling, etc.; works considering applications of fuzzy sets, fuzzy logics and related mathematical theories in other sciences: Medicine, Biology, Geology, Chemistry, etc.; works devoted to applications of fuzzy sets and rules of fuzzy logics in engineering and industry. These works differ very much not only in the subject of research, but also in their competence and quality: unfortunately along with deep, substantial research papers, there are also shallow, superficial papers, which, discredit the whole area among non-specialists in the Mathematics of fuzzy sets. It is impossible to give even a very brief objective survey on the most important works in the context of fuzzy sets which appeared in this period. In the next sections we shall discuss some of the works considering basic concepts as well as some aspects of the recent development of mathematics and other sciences appealing to fuzzy sets. The reader should be forewarned that this exposition in no case can be considered as representative and is dictated by the competence and the taste of the author.

\section{$3 \quad$ Fuzzy Sets}

\subsection{Fuzzy sets: basic concepts. $L$-fuzzy sets}

To grasp the idea of a fuzzy set note that a subset $A$ of a set $X$ can be described by its membership function $\chi_{A}: X \rightarrow\{0,1\}$ :

$$
\begin{cases}\chi_{A}(x)=1 & \text { if } x \in A \\ \chi_{A}(x)=0 & \text { if } x \notin A .\end{cases}
$$

Thus $\chi_{A}$ characterizes the degree to which an element $x$ belongs to a set $A$. This degree is either 0 or 1 . Now, allowing this degree to be also between 0 and 1 we come to Zadeh's concept of a fuzzy subset $A$ of a set $X$, denoted $A \tilde{\subset} X$ and characterized by its membership function $\mu_{A}: X \rightarrow[0,1]$. The value $\mu_{A}(x)$ is interpreted as the degree of belongness of a point $x \in X$ to a fuzzy set $A$.

Taking into account that fuzzy sets are "invented" it order to be a more satisfactory apparatus for the purposes of practice, and to reflect better the 
way of human reasonings, one can easily note that in some situations the unit interval $[0,1]$ as the codomain for membership functions is too restricted. We give an example of such situations. It concerns the fuzzy set $B$ of birds in the set of all living beings. Clearly a swallow and a nightingale belong to $B$ with degree 1, while a hippopotamus and a lion belong to it with degree 0 . But what about such "birds" as ostrich, penguin and kiwi? Ornithologists agree that all of them have some essential, but not all typical characteristics of birds. So we can interpret that a "bird" can belong to the "fuzzy set" $B$ of birds to a certain degree, between 0 and 1 . However characteristic bird traits of an ostrich, a penguin and a kiwi are different. Therefore one can hardly decide which of them belongs to $B$ to a larger extent. Hence we come to the idea that a membership function of a fuzzy set should be allowed to accept also incomparable values, (in this example incomparable values characterizing the belongness degree of an ostrich, a penguin and a kiwi to the fuzzy set $B$ of birds) between 0 (the bottom degree of belongness) and 1 (the maximal degree of belongness). This leads to the idea to consider fuzzy sets whose membership functions can accept incomparable values. This idea was developed by J.A. Goguen [22] and resulted in the concept of an $L$-fuzzy set. Namely, let $L$ be a complete lattice whose bottom and top elements are 0 and 1 respectively, see e.g. [9]. Further let $X$ be a set. Then an $L$-fuzzy subset $A \tilde{\subset} X$ is characterized by its membership function $\mu_{A}: X \rightarrow L$. In case $L=[0,1]$ an $L$-fuzzy set is just a fuzzy set $A \tilde{\subset} X$ as defined by L.A. Zadeh, and in case $L=\{0,1\}$ we actually redefine a usual subset $A$ of $X$.

At this point we note that generally $L$-fuzzy sets are not only more satisfactory for various applications, but also they are theoretically more interesting and promising for applications than its special kind, fuzzy sets as they were defined above. On the other hand the work in the context of general $L$-fuzzy sets is essentially more complicated than in the context of "ordinary" Zadeh's fuzzy sets, that is $L$-fuzzy sets in the special case when $L=[0,1]$. Therefore in the sequel we shall consider only the case of "ordinary" fuzzy sets.

When working with fuzzy sets a helpful tool is the so called level decomposition of a fuzzy set. Namely, the subsets $A_{\alpha}:=\left\{x \in X \mid \mu_{A}(x) \geq \alpha\right\}, \alpha \in L$, of the set $X$ are called levels of a fuzzy set $A \tilde{\subset} X$ and the family $\left\{A_{\alpha} \mid \alpha \in L\right\}$ is called the level decomposition of the fuzzy set $A$. One can easily see that $A_{0}=X$ and that $\left.\left.\bigcap_{\beta<\alpha} A_{\beta}=A_{\alpha} \forall \alpha \in\right] 0,1\right]$. Conversely, starting with a system of sets with such properties a corresponding fuzzy set $A$ can be reconstructed by setting $\mu_{A}(x)=\sup \left\{\alpha \in[0,1] \mid x \in A_{\alpha}\right\}$, having $A_{\alpha}$ as its level sets.

\subsection{Operations with fuzzy sets}

One of the first problems to be discussed when dealing with fuzzy sets is how the basic operations: intersection, union and complement with fuzzy sets should be defined. Although one can suggest several "natural" ways how to do this, ${ }^{2}$ the most often accepted is the following.

\footnotetext{
${ }^{2}$ Note that they must be mutually interrelated in the "natural way" and extend operations with ordinary sets!
} 
The intersection $A \tilde{\cap} B$ of fuzzy sets $A \tilde{\subseteq} X$ and $B \tilde{\subseteq} X$ is defined by the membership function $\mu_{A \tilde{\cap} B}=\min \left\{\mu_{A}, \mu_{B}\right\}\left(:=\mu_{A} \wedge \mu_{B}\right)$ (cf. with the crisp case $\left.\chi_{A \cap B}=\min \left\{\chi_{A}, \chi_{B}\right\}\right)$.

The union $A \tilde{\cup} B$ of these fuzzy sets is defined by the membership function $\mu_{A \tilde{}}=\max \left\{\mu_{A}, \mu_{B}\right\} \quad\left(:=\mu_{A} \vee \mu_{B}\right)$ (cf. with the crisp case $\chi_{A \cup B}=$ $\max \left\{\chi_{A}, \chi_{B}\right\}$.) The complement $A^{c}$ of a fuzzy set $A \subseteq X$ is defined by the membership function $\mu_{A^{c}}=1-\mu_{A}$ (cf. with $\chi_{A^{c}}=1-\chi_{A}$ ).

Given a mapping $f: X \rightarrow Y$, the membership function $\mu_{f(A)}: Y \rightarrow[0,1]$ of the image $f(A) \tilde{\simeq} Y$ of a fuzzy subset $A \widetilde{\simeq} X$ is defined by the equality

$$
\mu_{f(A)}(y)=\sup \{A(x) \mid f(x)=y\} \quad \text { where } \sup \emptyset:=0
$$

and the membership function $\mu_{f^{-1}(B)}: X \rightarrow[0,1]$ of the preimage $f^{-1}(B)$ of a fuzzy set $B \tilde{\subseteq} Y$ is defined by the equality

$$
\mu_{f^{-1}(B)}(x)=\left(\mu_{B} \circ f\right)(x)\left(=\mu_{B}(f(x))\right) .
$$

These definitions are also well coordinated with the definitions of images and preimages of usual sets when the last ones are rewritten in the form of the corresponding characteristic functions.

So thus defined operations with fuzzy sets are "natural" extensions of the corresponding operations with ordinary sets. Besides these definitions are wellgrounded from the theoretical (in particular, the categorical) as well as from the practical point of view. An interested reader can find much additional information in this concern, e.g. in [67] or in [14]. Most of the properties of these operations are natural analogues of the corresponding properties of their classical analogous. In particular, the operation of union and intersection are mutually distributive; the images and preimages of unions, intersections and complements behave themselves in the same way as in the case of usual sets.

An essential difference one first encounters when dealing with complementation of fuzzy sets: membership function $\mu_{A^{c} \tilde{\cap} A}$ of the intersection $A \tilde{\cap} A^{c}$ need not be $\mu_{\emptyset}$ and membership function $\mu_{A^{c} \tilde{\cup} \mathcal{A}}$ of the union $A \tilde{\cup} A^{c}$ need not be $\mu_{X}$.

Since fuzzy sets are defined by their membership functions, there arises a temptation to identify a fuzzy set $A \subseteq \tilde{\simeq}$ with the function $\mu_{A}: X \rightarrow[0,1]$, that is to write just $A: X \rightarrow[0,1]$. This is really convenient and such identification is done by many authors, especially by those working in the fields of pure mathematics. Also such perception of fuzzy sets is often accepted in this paper.

\subsection{Fuzzy points}

A special kind of fuzzy subsets of a set is chosen which can be interpreted as a fuzzy analogue of points. Namely, a fuzzy point in a set $X$ is a fuzzy set $x^{\alpha} \tilde{\subseteq} X$, where $x \in X$ and $\alpha \in(0,1]$ which is determined by the membership function $p^{\alpha}{ }_{x}: X \rightarrow L$ :

$$
p^{\alpha}{ }_{x}(y):= \begin{cases}\alpha & \text { if } y=x \\ 0, & \text { if } y \neq x\end{cases}
$$

Two relations of a fuzzy point $x^{\alpha}$ to a fuzzy set $A$ are used (see e.g. [62]): belongness relation $x^{\alpha} \tilde{\in} A$ if $\alpha \leq \mu_{A}(x)$ (analogue of crisp $x \in A$ ) and quasicoincidence relation $x^{\alpha} \tilde{q} A$ if $\alpha+\mu_{A}(x)>1$ (analogue of the crisp relation 
$x \notin X \backslash A)$. Obviously in the crisp case both relations coincide, while in the fuzzy case one has to choose which one of the two relations should be used in each particular situation.

Although fuzzy points in some situations can be used as right fuzzy substitutes for crisp (that is usual) points, their "nature" is essentially different from the "nature" of crisp points. In particular, while a crisp point is a minimal object (nothing can belong to a point!), this is not the case in the fuzzy situation: $x^{\alpha} \in x^{\beta}$ whenever $\alpha \leq \beta$. Also the behaviour of belongness and quasicoincidence relations of fuzzy points with respect to union and intersection of fuzzy sets essentially differs from the behavior of their crisp counterparts.

\section{Fuzzy Numbers}

One of the first problems which is encountered by everyone working with fuzzy sets, is what the fuzzy analogue of a real number should be and how the arithmetic operations with fuzzy numbers must be defined. In the literature one can find two essentially different approaches to the problem of a fuzzy real number. Both of them agree that a fuzzy real number should be a certain fuzzy subset of the real line $\mathbb{R}$. However they differ on specifying which fuzzy subsets of $\mathbb{R}$ should be considered as fuzzy numbers. While the first approach is based on understanding a fuzzy real number as a generalized interval in $\mathbb{R}$, the second one views a fuzzy number as a certain distribution-type function $z: \mathbb{R} \rightarrow[0,1]$. Concerning the definition of arithmetic operations with fuzzy real numbers the adherents of the both approaches agree that they should be defined on the basis of the Zadeh's extension principle: we shall illustrate this principle on the example of interval-type fuzzy numbers. Before giving a brief sketch of these approaches we note that the interval-type approach is supported mainly by application-oriented scientists, while the second is more justified from the theoretical point of view. It is also worth mentioning that although starting from essentially different ideas they are not completely unrelated (see e.g. [16]).

\subsection{Interval-type fuzzy numbers and Zadeh's extension principle}

The adherents of what we call "the interval-type approach", view fuzzy numbers as intervals with blurred borders. The idea of this approach stems from the early works of Zadeh, and later elaborated by different authors, in particular by M. Mizumoto and K. Tanaka [50]. According to the interval-valued approach a fuzzy number is a fuzzy set $A \tilde{\subset} \mathbb{R}$ which is defined by a convex membership function $\mu_{A}: \mathbb{R} \rightarrow$ I where the convexity means

$$
\min \left\{\mu_{A}\left(x_{1}\right), \mu_{A}\left(x_{3}\right)\right\} \leq \mu_{A}\left(x_{2}\right) \quad \text { whenever } x_{1} \leq x_{2} \leq x_{3},
$$

and hence the levels $A_{\alpha}$ of a fuzzy set $A \tilde{\subset} X$ are intervals, probably unbounded, in $\mathbb{R}$. Often it is additionally required that $\mu_{\alpha}$ should be upper-semicontinuous $[17,33]$ and that the closure of the support $\left\{x \mid \mu_{A}(x)>0\right\}$ should be compact. Let $\mathbb{E}$ denote the set of all such fuzzy numbers. A nice property of $A \in \mathbb{E}$ is that for all $\alpha>0 \alpha$-levels $A_{\alpha}$ are closed intervals in $\mathbb{R}$ and therefore a metric 
$D$ can be introduced on $\mathbb{E}$ by setting $D(A, B)=\sup _{\alpha>0} d\left(A_{\alpha}, B_{\alpha}\right)$, where $d$ is the Hausdorff metric $[17,33]$ on the set of all compact subsets of $\mathbb{R}$.

There are several special classes of fuzzy numbers of which we mention the triangular fuzzy numbers $A:=(a, b, c)$. These fuzzy numbers are characterized by membership functions defined by the equation

$$
\mu_{A}(x)= \begin{cases}0 & \text { if } x \leq a, \\ \frac{x-a}{b-a} & \text { if } a<x \leq b, \\ \frac{x-c}{b-c} & \text { if } b \leq x<c \\ 0 & \text { if } c \leq x\end{cases}
$$

In order to consider the case when some of the equalities $a=b=c$ hold we have to make specification that also in this case $\frac{b-a}{b-a}=1, \frac{b-c}{b-c}=1$. Thus, if $a=b=c$, then the fuzzy number $(a, a, a)$ can be identified with a real number $a \in \mathbb{R}$. Having fuzzy numbers in mind, one starts to wonder how to operate with them. In other words what is the arithmetics of fuzzy numbers? And here the appropriate way is to use the s.c. extension principle [94]. In its simplest way it looks as follows: Given an operation $\varphi: \mathbb{R} \times \mathbb{R} \rightarrow \mathbb{R}$ one extends it to the operation on the set $\mathbb{E}$ of all fuzzy numbers by setting

$$
\tilde{\varphi}\left(\mu_{A}(x), \mu_{B}(x)\right)=\sup \left\{\mu_{A}(s) \wedge \mu_{B}(t): \varphi(s, t)=x\right\} .
$$

In particular $\left(\mu_{A+B}\right)(x)=\sup \left\{\mu_{A}(s) \wedge \mu_{B}(t) \mid s+t=x\right\}$ and $\left(\mu_{A \cdot B}\right)(x)=$ $\sup \left\{\mu_{A}(s) \wedge \mu_{B}(t) \mid s \cdot t=x\right\}$.

Note that when restricted to (ordinary) real numbers these operations reduce to the usual arithmetic operations. However the arithmetics of fuzzy numbers is much more complicated than ordinary arithmetics, in particular what concerns inverse operations like subtraction and division.

Recently some papers were published where sequence spaces of fuzzy numbers where studied and used. In particular the authors of $[18,19,52,87]$ under additional assumptions investigate the statistical-type convergences in difference sequence spaces of fuzzy numbers.

\subsection{Fuzzy numbers as distribution-type functions and the fuzzy real line}

Along with interval-type fuzzy numbers, the idea to interpret fuzzy numbers as certain distribution-type functions is very popular in "fuzzy mathematics". Probably the first works where this idea is traced are [29] and [20]. Slightly modifying the definitions introduced in $[20,29]$ we define a fuzzy number as a non-increasing upper-semicontinuous (see e.g. [17, 33]) function $z: \mathbb{R} \rightarrow[0,1]$ such that $\sup z(x)=1$ and $\inf z(x)=0$. An ordinary number $a \in \mathbb{R}$ in this approach is identified with the fuzzy number

$$
z_{a}(x)= \begin{cases}1 & \text { if } x \leq a \\ 0, & \text { if } x>a\end{cases}
$$

The set of all fuzzy numbers is called the fuzzy real line and is denoted $\mathbb{R}([0,1])$. The arithmetics of such fuzzy numbers is also defined by the extension principle. 
This arithmetic, as well as the topological structure of $\mathbb{R}([0,1])$ was thoroughly studied by S.E. Rodabaugh [66]. Fuzzy real numbers defined in this way are useful, in particular, in fuzzy topology see e.g. [29, 30, 66, 83], etc., theory of fuzzy measures see e.g. $[38,72,73]$, approximation theory under imprecise information, see $[6,7]$, etc. In the sequel we will refer also to the subset $\mathbb{R}^{+}([0,1])$ of $\mathbb{R}([0,1])$ consisting of all of all non-negative fuzzy numbers that is fuzzy numbers $z: \mathbb{R} \rightarrow[0,1]$ such that $z(0)=1$.

A deep theoretical justification of viewing fuzzy numbers as distributiontype functions was given by U. Höhle [27], who showed that such fuzzy real numbers can be obtained from the set of rational numbers $\mathbb{Q}$ by means of Dedekind completion in the same way as (ordinary) real numbers $\mathbb{R}$ are obtained from $\mathbb{Q}$ if one applies the Eukasiewicz logic (see 5.2), instead of the binary logic which stands behind the Dedekind completion in the classic case.

\section{$5 \quad$ Fuzzy Logics}

\subsection{Connectives in classical logic}

To give a reader insight into fuzzy logics we start by analyzing basic connectives of the classical logic - conjunction, disjunction, implication, and negation. Usually they are given in the form of so called tables of truth values:

\begin{tabular}{|llll|}
\hline$\&$ & 0 & 1 \\
\hline 0 & 0 & 0 \\
\hline 1 & 0 & 1 \\
\hline
\end{tabular}

Conjunction \&

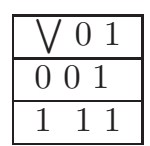

Disjunction $\bigvee$

\begin{tabular}{|llll|}
\hline$\mapsto$ & 0 & 1 \\
\hline 0 & 1 & 1 \\
\hline 1 & 0 & 1 \\
\hline
\end{tabular}

Implication $\mapsto$



Negation $\neg$

Thus connectives in classical logic can be interpreted as mappings describing how truth values of original propositional statements are reflected in the truth value of the resulting propositional statement:

$$
\&, \bigvee, \mapsto:\{0,1\} \times\{0,1\} \rightarrow\{0,1\}, \quad \neg:\{0,1\} \rightarrow\{0,1\}
$$

\subsection{Connectives in fuzzy logics}

In fuzzy logics propositions $P$ can be not only true or false but also true and false to a certain degree: $t(P) \in[0,1]$. Thus connectives of fuzzy logics must be also functions describing how truth values of original propositional statements are reflected in the truth value of the resulting propositional statement:

$$
\&, \bigvee, \mapsto:[0,1] \times[0,1] \rightarrow[0,1], \quad \neg:[0,1] \rightarrow[0,1]
$$

There are various "natural" ways how these connectives could be defined. 3 However one has to care that the following two principles should be satisfied. First, when restricted to the range $\{0,1\} \subset[0,1]$ of the connectives, the truth value tables of classical logic should be received, and second, the connectives

\footnotetext{
${ }^{3}$ Just for this reason we use the word fuzzy logics in plural.
} 
of fuzzy logics should me mutually interrelated in the way, analogous to the mutual interrelations of connectives of classical logic. The fuzzy negation $\neg$ most often is given by the usual subtraction in the unit interval: $\neg \alpha=1-\alpha$, $\forall \alpha \in[0,1]$. On the other hand conjunction can be defined in different "natural" ways what in its turn reflects in the definitions of the corresponding disjunction and implication, and, in the long run, results in different logics. We consider the three most popular ones. Note that the three important $t$-norms (see e.g. [36]) make the background for these logics.

- Fuzzy conjunction $\&:[0,1] \times[0,1] \rightarrow[0,1]$ based on the minimum $t$-norm:

$$
\&(\alpha, \beta)=\alpha \wedge \beta
$$

the corresponding disjunction and implication are given, respectively, by $\bigvee(\alpha, \beta)=\alpha \vee \beta$ and

$$
\mapsto(\alpha, \beta)= \begin{cases}1 & \text { if } \alpha \leq \beta \\ \beta, & \text { if } \alpha<\beta\end{cases}
$$

- Fuzzy conjunction \& : $[0,1] \times[0,1] \rightarrow[0,1]$ based on the Eukasiewicz $t$-norm:

$$
\&(\alpha, \beta)=\max \{\alpha+\beta-1,0\} ;
$$

the corresponding disjunction and implication are given, respectively, by $\bigvee(\alpha, \beta)=\min \{\alpha+\beta, 1\}$ and $\mapsto\{\alpha, \beta\}=\max \{1-\alpha+\beta, 0\}$.

- Fuzzy conjunction $\&:[0,1] \times[0,1] \rightarrow[0,1]$ based on the product $t$-norm:

$$
\&(\alpha, \beta)=\alpha \cdot \beta
$$

the corresponding disjunction and implication are given respectively by $\bigvee(\alpha, \beta)=\alpha+\beta-\alpha \cdot \beta$ and

$$
\mapsto(\alpha, \beta)= \begin{cases}\beta / \alpha & \text { if } \beta \leq \alpha \text { and } \alpha \neq 0, \\ 1 & \text { if } \beta>\alpha \text { or } \alpha=0 .\end{cases}
$$

\subsection{Remark}

It is important to make some remarks concerning the term "Fuzzy Logic". Different authors interpret this term in a different way. In the literature interpretations of the term "Fuzzy Logic" are usually divided into two large groups: Fuzzy Logic in the broad sense (more in the spirit of applied mathematics) and Fuzzy Logic in the narrow sense (more in the spirit of theoretical mathematics). These groups are "fuzzy" in the sense that there are many "overlapping" cases. According to this division, the fuzzy logics considered in Section 5.2 belong to the group of Fuzzy Logic in the narrow sense. An interested reader can find detailed information about different interpretations of the concept of a Fuzzy Logic in monographs $[24,54,55,56]$. 


\section{Mathematical Structures on the Basis of Fuzzy Sets}

As it has been already mentioned, since the middle seventies of the $19^{\text {th }}$ century counterparts of various areas of traditional mathematics are being developed in the context of fuzzy sets. In this paper we shall discuss four of these areas: topology (which is the author's main subject of research), differential calculus, differential equations, and theory of measure and integral. As the reader will see from these examples, there are several different natural ways how to develop counterparts of traditional mathematical areas in the context of fuzzy sets. To make our exposition more clear and visual in the sequel we identify a fuzzy set $A \tilde{\subset} X$ with its membership function $\mu_{A}: X \rightarrow[0,1]$ see Section 3.2 ; in particular an ordinary subset $A \subset X$ is identified with its membership function $\chi_{A}: X \rightarrow\{0,1\}$, and hence the empty set $\emptyset$ and the universe set $X$ are identified with constant functions $\chi_{\emptyset}=0_{X}$ and $\chi_{X}=1_{X}$ respectively. The union and the intersection of fuzzy sets $A, B: X \rightarrow[0,1]$ in this case are identified with the supremum and infimum of the corresponding membership functions $A \vee B: X \rightarrow[0,1]$ and $A \wedge B: X \rightarrow[0,1]$ respectively.

\subsection{Topological structures on the basis of fuzzy sets}

\subsubsection{Topological structures on families of fuzzy sets}

The first approach to topology in fuzzy setting (and probably also the first attempt to consider any mathematical structure in the context of fuzzy sets) was proposed in 1968 by C.L. Chang, [12], a student of L.A. Zadeh. According to Chang, a fuzzy topology on a set $X$ is a family of its fuzzy subsets, i.e. $\tau \subseteq[0,1]^{X}$, such that

1. $0_{X}, 1_{X} \in \tau$ (cf. the topological axiom $\left.\emptyset \in \tau, X \in \tau\right)$;

2. $U, V \in \tau \Rightarrow U \wedge V \in \tau$ (cf. the topological axiom $U, V \in \tau \Rightarrow U \cap V \in \tau$ );

3. $U_{i} \in \tau \forall i \in I \Rightarrow \bigvee_{i} U_{i}$ (cf. the topological axiom $U_{i} \in \tau \forall i \in I \Rightarrow$ $\left.\bigcup_{i} U_{i} \in \tau\right)$.

Given two fuzzy topological spaces $\left(X, \tau_{X}\right),\left(Y, \tau_{Y}\right)$, a mapping $f:\left(X, \tau_{X}\right) \rightarrow$ $\left(Y, \tau_{Y}\right)$ is called continuous if $V \in \tau_{Y} \Rightarrow f^{-1}(V) \in \tau_{X}$. Soon this definition was extended by J.L. Goguen [23] to the case of $L$-fuzzy topological spaces where $L$ is a complete infinitely distributive lattice by replacing the family of fuzzy sets $[0,1]^{X}$ with the family of $L$-fuzzy sets $L^{X}$.

Chang-Goguen's definition could seem to be the only "natural" one extension of topology to the fuzzy case. However the properties of fuzzy topologies defined in this way have several essential differences from the properties of ordinary topologies. One of the most important distinctions is that constant mappings of fuzzy topological spaces need not be continuous. ${ }^{4}$ As consequences of this fact follow that the projections in the products of fuzzy topological spaces need not be open, a product of fuzzy topological spaces need not have

\footnotetext{
${ }^{4}$ We do not argue whether it is a strong or a weak point of the theory - the opinion of different authors vary in this respect.
} 
subspaces homeomorphic to its factors, etc. In order to "remedy" this "deficiency" R. Lowen [46] suggested to redefine the first axiom by requesting that all constant functions $\alpha_{X}$ and not only $0_{X}$ and $1_{X}$ should belong to $\tau$ :

$$
1^{\prime} . \alpha_{X} \in \tau \forall \alpha \in[0,1] \text {. }
$$

Now fuzzy topologies satisfying axiom $1^{\prime}$ are called stratified.

\subsubsection{Fuzzy topological structures on families of fuzzy sets}

In the previous approaches to the concept of a topology in the context of fuzzy sets only "sets" were fuzzy, but the "topology" was actually crisp, non fuzzy: it was a usual subset $\tau$ of the family of fuzzy sets $[0,1]^{X}$. Viewing this as a certain inconsistency of interpretation of topology in the context of fuzzy sets, the author of this paper [82] and T. Kubiak [40] (independently) developed a more general concept of a fuzzy topology. Namely according to [40, 82] a fuzzy topology on a set $X$ is a fuzzy family $\mathcal{T}:[0,1]^{X} \rightarrow[0,1]$ such that

1. $\mathcal{T}\left(0_{X}\right)=\mathcal{T}\left(1_{X}\right)=1$;

2. $\mathcal{T}(U \wedge V) \geq \mathcal{T}(U) \wedge \mathcal{T}(V) \forall U, V \in F(X)$

3. $\mathcal{T}\left(\bigvee_{i} U_{i}\right) \geq \bigwedge_{i} \mathcal{T}\left(U_{i}\right)$ for every family $\left\{U_{i} \mid i \in \mathcal{I}\right\} \subseteq F(X)$.

Given two fuzzy topological spaces $\left(X, \mathcal{T}_{X}\right),\left(Y, \mathcal{T}_{Y}\right)$ a mapping $f:\left(X, \mathcal{T}_{X}\right) \rightarrow$ $\left(Y, \mathcal{T}_{Y}\right)$ is called continuous if $V \leq f^{-1}(V) \forall V \in[0,1]^{X}$.

\subsubsection{Fuzzy topologies as subsets of lattices and variable-basis fuzzy topologies}

A principally different and quite interesting, view on the subject of Fuzzy Topology was proposed by B. Hutton [30] who interpreted fuzzy topologies as certain subsets of completely distributive lattices. Hutton's idea was later developed into the so called variable-basis fuzzy topology by S. Rodabaugh, see [65], etc.

An interested reader can find a detailed survey of these and some other approaches to the interpretation of topological structures in the context of fuzzy sets in our works $[28,83,84]$.

\subsection{Differential calculus in the context of fuzzy sets}

There are many works where problems of differentiation in the context of fuzzy sets are studied. However the specification of what should be the subject of the study, that is "what" and "in what sense" should be fuzzy, vary in different works. To give a reader insight of what could be the subject of "fuzzy differentiation" we briefly describe two approaches to this problem.

\subsubsection{Derivative of a fuzzy set}

One of the first attempts to study the problem of differentiation in the context of fuzzy sets was undertaken by D. Dubois and H. Prade in [15]. Following 
these works we illustrate how derivative of a function at a fuzzy set could be defined.

Let $f:[a, b] \rightarrow \mathbb{R}$ where $[a, b] \subset \mathbb{R}$ be a differentiable mapping and let $f^{\prime}$ be its derivative. Further, let $A \tilde{\subset}[a, b]$ be a fuzzy subset of $\mathbb{R}$ whose membership function $\mu_{A}$ is convex (see Section 4.1). The derivative of $f$ at the fuzzy set $A$ is defined as the fuzzy set $f^{\prime}(A)(y)=\sup \left\{A(x) \mid x \in f^{\prime-1}(y)\right\}$, that is as the image of the fuzzy set $A$ under mapping $f^{\prime}$. The value $f^{\prime}(A)$ is interpreted as the fuzzy set of the possible values of the derivative $f^{\prime}$ at a point $x \in \mathbb{R}$ whose possible positions are regulated by the fuzzy set $A$. One can see that $f^{\prime}(A)$ is also determined by a convex membership function $\mu_{f^{\prime}(A)}$. D. Dubois and H. Prade interpret this definition of a derivative at a fuzzy set by saying "the uncertainty about the precise location of the point whose position is only roughly specified by $A$ induces an uncertainty about the value of the derivative of $f$ at this point." Note that if $f^{\prime}(x)$ is constant on the support $\{x \mid A(x)>0\}$ of $A$, then $f^{\prime}(P)$ is a crisp set. In particular, $f^{\prime}(A)=f^{\prime}(x)$ in case $A$ is a crisp point, that is $A=(x, x, x)$ in notations of Section 4.1 Concerning linearity of differentiation it is shown that $f^{\prime}(A)+g^{\prime}(A) \geq\left(f^{\prime}+g^{\prime}\right)(A)$ and, in case the derivatives $f^{\prime}$ and $g^{\prime}$ are continuous and both nondecreasing or nonincreasing $f^{\prime}(A)+g^{\prime}(A)=\left(f^{\prime}+g^{\prime}\right)(A)$. Also an analogue of the rule of differentiation of the product holds:

$$
(f \cdot g)^{\prime}(A) \leq f^{\prime}(A) \cdot g(A)+f(A) \cdot g^{\prime}(A)
$$

the equality is fulfilled, in particular, in case when $f$ and $g$ are positive and $f^{\prime}$ and $g^{\prime}$ are both nondecreasing.

\subsubsection{Differentiation of fuzzy-valued mappings}

An alternative viewpoint on the problem of differentiation in the context of fuzzy sets was developed by M.L. Puri an d D.A. Ralescu [63], it generalizes Hukahara's differentiability of set-valued mappings. A mapping $F:[a, b] \rightarrow \mathbb{E}$ (see 4.1 for the definition of $\mathbb{E}$ ) is called differentiable at a point $t_{0} \in[a, b]$ if there exists a $F^{\prime}\left(t_{0}\right) \in \mathbb{E}$ such that the limits

$$
\lim _{h \rightarrow 0^{+}} \frac{F\left(t_{0}+h\right)-F\left(t_{0}\right)}{h} \text { and } \lim _{h \rightarrow 0^{+}} \frac{F\left(t_{0}\right)-F\left(t_{0}-h\right)}{h}
$$

exist and are equal. These limits are taken in the metric space $(\mathbb{E}, D) 4.1$, and the difference $x-y$ in $\mathbb{E}$ is defined as an element $z \in \mathbb{E}$ such that $x=y+z$.

Basic properties of such derivatives are considered in [63] and in [31]. In particular, it is shown that a differentiable function is continuous. If $F:[a, b] \rightarrow$ $\mathbb{E}$ is differentiable and $t_{1} \leq t_{2} \in[a, b]$, then there exists $C \in \mathbb{E}$ such that $F\left(t_{2}\right)=F\left(t_{1}\right)+C$, and hence for each $\alpha \in[0,1]$ the function $t \rightarrow \operatorname{diam}[F(t)]_{\alpha}$ is nondecreasing on $[a, b]$. The operation of differentiation behaves "in the natural way": If functions $F, G:[a, b] \rightarrow \mathbb{E}$ are differentiable and $\lambda \in \mathbb{R}$, then $(F+G)^{\prime}(t)=F^{\prime}(t)+G^{\prime}(t)$ and $(\lambda F)^{\prime}(t)=\lambda F^{\prime}(t)$.

Applying the construction of an integral of a fuzzy-valued function defined by Puri and Ralescu [63], O. Kaleva [31] shows that if $F:[a, b] \rightarrow \mathbb{E}$ is continuous, then for all $t \in[a, b]$ the integral $G(t)=\int_{a}^{t} F(t) d t$ is differentiable and 
$G^{\prime}(t)=F(t)$. A fuzzy version of Newton-Leibniz's formula is also established:

$$
F(s)=F(a)+\int_{a}^{s} F^{\prime}(t) d t .
$$

\subsection{Fuzzy differential equations}

Different versions of differential equations, in particular, of the initial value problem, in the fuzzy context were studied in several papers. For example O. Kaleva [31] studies the problem

$$
x^{\prime}(t)=f(t, x(t)), \quad x(a)=x_{0},
$$

where $f:[0,1] \times \mathbb{E} \rightarrow \mathbb{E}$ is a continuous function and shows that if $f$ satisfies an analogue of the Lipshiz condition $D(f(t, x), f(t, y)) \leq k D(x, y)$ for some constant $k>0$ and for all $t \in[a, b], x, y \in \mathbb{E}$, then this problem has a unique solution on $[a, b]$. O. Kaleva studies the "Fuzzy" Cauchy problem also in [32]

Discussing the actuality of the study of the initial value problem in the context of fuzzy sets Seikalla [74] writes: "When a physical problem is transformed into a deterministic initial value problem we usually cannot be sure that the modeling is perfect. The initial value may not be known exactly and the function $f$ may contain unknown parameters. Especially, if they are known through some measurements they necessarily are subjected to errors... If the nature of errors is random, then instead of deterministic problem we get a random differential equation... But if the underlying structure is not probabilistic, e.g. because of subjective choices then it may be appropriate to use fuzzy numbers instead of random variables."

\subsection{Measure and integral}

The author has found it especially difficult to make a brief unbiased survey of the works where measure and integral in the context of fuzzy sets are considered. The main problem here is a very large number of works dealing with these concepts and the principal diversity of interpretations of what should or could be the subject of the research. Probably the main reason why so much interest in this subject was expressed is that measures and integrals in the context of fuzzy sets have found applications in several applied sciences, in particular in mathematical economics, in optimization problems and control theory. So the permitted extend of this paper allowed the author just to point out only some of the approaches to this problem. Much more information and a vast list of references on this topic one can find in $[11,38]$ and in [45].

1. Probability measures of fuzzy events and fuzzy probability measures. Let $(\Omega, \mathcal{A}, P)$ be a probability space and let $\mathcal{F}:=\{\varphi: \Omega \rightarrow$ $[0,1] \mid \varphi$ is $A$-measurable $\}$ be interpreted as the collection of fuzzy events. L.Zadeh [95] defined probability measure of fuzzy events as the mapping $m: \mathcal{F} \rightarrow[0,1]$ determined by $m(\varphi)=\int_{\Omega} \varphi d P$ where on the right-hand side is the Lebesgue integral. In [37] the construction of a probability measure of fuzzy events is generalized to the construction of a fuzzy probability measure $m: \mathcal{F} \rightarrow[0,1]$ where $\mathcal{F}$ is a $\sigma$-algebra of fuzzy sets. 
2. Monotone fuzzy measure and Sugeno fuzzy integral. M. Sugeno [86] defines the notion of a fuzzy measure by replacing the requirement of $\sigma$-additivity in the definition of an (ordinary) measure by a weaker requirement of monotonicity $(m(A) \leq m(B)$ whenever $A \subseteq B)$ and continuity from below $\left(\left(A_{n}\right)_{n \in \mathbb{N}} \nearrow A \Longrightarrow \lim _{n \rightarrow \infty}=m(A)\right)$ and from above $\left(\left(A_{n}\right)_{n \in \mathbb{N}} \searrow A \Longrightarrow \lim _{n \rightarrow \infty}=m(A)\right) .^{5}$ In the same work Sugeno uses this fuzzy measure to define integral called now by his name. Later Sugeno fuzzy measures and integrals as well as their "fuzzified" versions were studied and applied by many authors, see e.g. [64].

3. (Fuzzy) possibility measures. A (fuzzy) possibility measure, see e.g. [96] is a mapping $m: \mathcal{F} \rightarrow[0,1]$ where $\mathcal{F}$ is a $\sigma$-algebra of either crisp or fuzzy sets, which is continuous from below and $m(A \wedge B)=$ $\max (m(A), m(B))$ for any $A, B \in \mathcal{F}$.

4. Triangular norm-based measures. Efforts of some researchers, were directed to the study of triangular norm-based measures in the definition of which instead of intersection and union a $t$-norm and the corresponding $t$-co-norm are used. In particular, (fuzzy) possibility measures can be described as $\wedge$-normed measures where $\wedge$ is the minimum $t$-norm.

5. Fuzzy-valued measures and fuzzy-valued fuzzy measures: Measures with values in the collection $\mathcal{D}_{\infty}$. E.P. Klement [35] considers measures $m: \mathcal{F} \rightarrow \mathcal{D}_{\infty}$ where $\mathcal{F}$ is a sigma-algebra of fuzzy sets and $\mathcal{D}_{\infty}$ is the collection of all probability distribution functions on $[0, \infty)$.

6. Fuzzy-valued measures and fuzzy-valued fuzzy measures: Measures with values in the set interval-type fuzzy numbers $\mathbb{E}$. Measures with values in the set $\mathbb{E}$ of of interval-type fuzzy numbers were considered in a series of papers by Hsien-Chang Wu. In particular, in [92] the foundations of the theory of fuzzy-valued integrals of fuzzy-valued measurable functions with respect to $\mathbb{E}$-valued measures is being developed.

\section{Applications of Fuzzy Sets and Fuzzy Logics in Other Sciences and in Industry}

\subsection{Mathematical modelling}

A vast research work was done in the field of mathematical modelling on the basis of fuzzy sets and fuzzy logics. An interested reader can get a good conception about problems studied in the frames of fuzzy modeling from the monograph "Fuzzy modeling: paradigms and practice" edited by one of the founders and actively working in this field American scientist of Polish descent Wytold Pedricz [59]. Here we give two quotations from [59] which, in our opinion, give

\footnotetext{
${ }^{5}$ Actually the idea to consider monotone non-additive measures was first suggested by G. Choquet in his theory of capacities. Besides we pay the readers' attention that the term fuzzy measure in this case is somewhat misleading since actually there are no fuzzy sets involved in its definition. However for historical reasons many researchers continue to call it a fuzzy measure.
} 
a concise but precise idea about this field:

"The essence of fuzzy modeling is concerned with constructing models that flexibly core heterogeneous data including those of linguistic and numerical characters", "Briefly speaking, fuzzy models are modeling constructs flattering two main properties: they operate at the level of linguistic terms (fuzzy sets); similarly all system dependences can be portrayed in the same linguistic format and they represent the process uncertainty."

\subsection{Fuzzy sets and fuzzy logics in other sciences}

The number of works where fuzzy sets and reasonings on the basis of fuzzy logics are applied grow in the last decades in the avalanche way. Although it is impossible to give a representative survey of these works, here we shall mention only some of them, which reflect the author's interests.

In 2004 a monograph "Fuzzy Logic in Geology" [13] was published. This book consists of 10 Chapters written by different authors, among them "Fuzzy logic and Earth Sciences", "Fuzzy logic in Geological Sciences" by R.V.Demicco, "Formal concept analysis in Geology" by Belohlavek, "Fuzzy logic and Earthquake research" by C. Huang, "Fuzzy transform: application to the reef growth problem" by I. Perfilieva and "Ancient sea level estimation" by V. Novak.

Much work has been done in the field of applications of fuzzy sets and rules of fuzzy logic in life sciences - biology and medicine. The information available to the physician about his patient and about medical relationships in general is inherently uncertain. Nevertheless, the physician is still capable to make approximate conclusions from this information. Fuzzy set theory makes it possible to interpret inexact medical considerations as fuzzy sets, thus creating a method of "translating" intuitive, to a certain extent, reasonings of a physician into formal mathematically grounded conclusions.

To justify the appropriateness of methods based on fuzzy sets in medical sciences K.P. Adlassing [1] mentions some properties of fuzzy set theory allowing to formalize uncertain information upon which medical diagnosis and treatment is usually based. In particular, he notes that inexact medical entities could be formalized as fuzzy sets and that fuzzy sets provide a linguistic approach with "an excellent approximation to texts". Besides he notes that fuzzy logic "offers reasoning methods capable of drawing approximate inferences." Thus fuzzy set theory provides "a suitable basis for the development of a computerized diagnosis system." K.P. Adlassing has substantiated these considerations by tests performed with the medical expert system CADIAG-2 which uses fuzzy set theory to formalize medical relationships and fuzzy logic to model the diagnostic process.

An interested reader can find much information about the use of fuzzy sets in biology and medicine from the survey paper [2]. This work contains also an extended list of references to related papers. 


\subsection{Fuzzy sets and fuzzy logics in industry}

Starting with the end of eighties of the $20^{t h}$ century and especially in the last decade fuzzy sets, or more precisely, rules based on the laws of Fuzzy Logic are successfully used in industry. In the initial period Japanese and South Korean companies started to use them when designing the industrial process, while western companies remained restrained and started to realize the importance of the fuzzy rules much later. Some experts even affirm that one of the main reasons of the so called South Korean miracle (an extremely fast industrial development in the last decade) is the reconstruction of many branches of its economy on the basis of fuzzy rules. Here we shall present a small number of examples where fuzzy rules are used in industry. ${ }^{6}$

Mitsubishi produces air-conditioners using fuzzy rules in order to prevent overshoot-undershoot temperature oscillation and consume less on-off power. Nissan produces anti-lock brakes where fuzzy rules are used to control brakes in hazardous cases based on car and wheel speed and acceleration. In the washing machines produced by Daewoo and Samsung fuzzy rules are used to adjust washing strategy based on sensed dirt level, fabric type, load size and water level. Rice cooker (remember how important is rice in Asian cousine!) produced by Matsushita regulates by fuzzy rules cooking time and method based on steam, temperature and rice volume. Vacuum cleaners produced by Hitachi and Toshiba apply fuzzy rules in order to set motor-suction strategy based on dust quantity and floor type. Basing on fuzzy rules video cameras produced by companies Canon and Sanyo adjust autofocus and lighting as well as cancel hand trembling. LG and Samsung developed television systems using fuzzy rules to adjust screen colour and texture and to stabilize volume based on viewer's room location. Shower systems produced by Panasonic use fuzzy rules in order to suppress variation in water temperature.

Especially we would like to mention the work of professor of Tokyo Institute of Technology M. Sugeno, whose PhD Thesis [86] was one of the first important works in the field of pure mathematics in the context of fuzzy sets and who, on the other hand, is well-known as a specialist in applications of fuzzy sets in industry. M. Sugeno has built a system that can stabilize a helicopter in flight when it looses a rotor blade; this system uses about 100 fuzzy rules.

\section{Mathematics of Fuzzy Sets in Latvia}

Introductory remark. In this section we give a brief survey of the work in the field of "fuzzy mathematics" carried out in Latvia. The diversity of the directions in which this work was carried out as well as the desire to outline a representative part of this paper did not allow us to be as systematic as we tried to be in the previous parts of the work. In particular, many concepts used here remain undefined - an interested reader can find them in the corresponding references. Besides, here we often use a prefix $L$ when speaking about different types of fuzziness. Although it is quite important in many cases, to get just

\footnotetext{
${ }^{6}$ The material presented in this section is taken mainly from the book [39] and updated from the Internet resources.
} 
an idea of what we are speaking about, a reader can omit this prefix and read just fuzzy set instead of $L$-fuzzy set, etc.

The starting point of interest in mathematical structures in the context of fuzzy sets in Latvia was in the middle of 1980's when the first works in fuzzy topology were published by the author of this paper, see e.g. [82, 83], etc. Among other in these works the concept of an L-fuzzy topology on a family of L-fuzzy sets 6.1.2 was introduced and the foundations of the theory of such spaces were laid. Later this theory was developed in many publications both in case when the base $L$ is fixed and in case when the base is changing, see e.g. [84]. Also the uniform and proximity counterparts of $L$-fuzzy topological structures on $L$-fuzzy sets were considered in the papers of the author of this work and coauthors, see e.g [21, 48, 84].

More than ten years have passed before a group of young Latvian researchers - A. Grasmanis, Z. Diskin, I. Uljane, I. Zvina, L. Drava et. al. showed interest in the problems of fuzzy topology. I. Ulane in a series of papers see e.g. $[90,91]$ and especially in her PhD Thesis [88] developed foundations of the theory of $L$-fuzzy topologies on many-valued sets. I. Zvina started to study $L$-fuzzy type generalization of the concept of a topological space via ideal [97, 98]. Later the idea of this concept was developed into a very fruitful theory discovering important relations between topology, lattices and locales [99, 100].

Since the end of nineties the scientific interests of our group was drawn also to the categorical aspects of mathematics in the context of fuzzy sets. Speaking about this area we have to separate two essentially different, although mutually related trends. One trend follows the concept of a fuzzy category, the ideas of which originate in my paper [82] and which much later were developed into a theory of $L$-valued categories. In this category either objects or morphisms or both are allowed to be such (that is objects and morphisms) only to a certain degree, see e.g. [80, 81]. Just this is the subject of O. Grigorenko's work [26], where the author constructs and studies an L-valued category whose objects are fuzzy ordered L-valued sets and morphisms are potential orderpreserving mappings (in a fuzzy sense). Also in I. Uljanes work [89] a fuzzy category of many valued sets and topological spaces is introduced and studied. On the other hand a large work done by S.Solovjov in his PhD Thesis (2007) [79] and in more than a dozen works published in the last 5 years see e.g. $[75,76,77,78]$. He deals with usual categories whose objects are $L$-fuzzy sets, $L$-fuzzy topological spaces, $L$-fuzzy algebraic structures and other systems of $L$-fuzzy objects. Also in the works by I. Uljane $[90,91]$ and her PhD Thesis [88] properties of a classical category of $L$-fuzzy subsets on many valued sets are studied.

Starting with the beginning of the last century the spectrum of interests of Latvian mathematicians in the field of fuzzy sets included also the theory of Measure and Integral and now important work is done in this field by S. Asmuss and V.Ruža, see e.g. [71, 72, 73]. We already mentioned the work done in the field of fuzzy measure and integral in 6.2. However, as different from the approaches considered in 6.2 the main interest of S.Asmuss' group is in developing a theory of measure and integral where not only sets are fuzzy, but also measure and integral take fuzzy real values. To reach these purposes 
they use $L$-fuzzy real numbers in Hutton's sense that is as they are defined in Section 4.2. The works of this group present a construction of an $L$-fuzzy valued $T$-measure by extending a measure defined on a $\sigma$-algebra of crisp sets to an $L$-fuzzy valued measure defined on a $T$-tribe of $L$-fuzzy sets in case when operations for $L$-fuzzy sets and $L$-fuzzy real numbers are based on a $t$-norm $T$ and $L$ is a completely distributive lattice. An $L$-fuzzy valued $T$-integral over an $L$-fuzzy set with respect to an $L$-fuzzy valued $T$-measure is constructed, its basic properties are investigated and a method for calculation is given.

After participating at the conference FSTA (Fuzzy Sets - Theory and Applications) in Slovakia in 2008, the interest of several younger members of the Latvian group was drawn to the theory of fuzzy aggregation operators. The most active among them are J. Lebedinska, O. Grigorenko and P. Orlovs. J. Lebedinska $[41,42,44]$ introduces and studies the operation of generalized aggregation. The term "generalized" refers to the generalized input and output of aggregation operators (agops), that is aggregation operators which aggregate fuzzy sets. J.Lebedinska develops two construction methods of generalized agops, namely a pointwise extension and a $T$-extension. When studying the properties of generalized aggregation, the crucial information is contained in two transitive order relations introduced by the author: vertical and horizontal order relations. J. Lebedinska studies how properties of an agop $A$ are reflected in the properties of its pointwise extension and its $T$-extension. O. Grigorenko $[25,26]$ introduces and studies the notion of degree of monotonicity for aggregation function. Namely, a fuzzy order relation is introduced in the definition of monotonicity for aggregation function. This relation allows to measure the degree to which a function is monotone: in its turn a fuzzy order relation allows to generalize the notion of monotonicity for aggregation processes. Properties of aggregation functions having a certain degree of monotonicity are studied.

The work of one of our youngest participants P. Orlovs in collaboration with S.Asmuss deals with arithmetic of $L$-fuzzy numbers (see Section 4.2) [57]. This arithmetic is based on a general aggregation operator introduced by $\mathrm{J}$. Lebedinska and is defined by using a $t$-norm $T$ as a $T$-extension of an ordinary aggregation operator and acts on $L$-fuzzy real numbers when $L$ is a completely distributive lattice. The main aim of this research is to analyze properties of this general aggregation operator depending on the properties of the ordinary aggregation operator and the underlying $t$-norm. By using aggregation approach some $t$-norm based operations with $L$-fuzzy real numbers are described and their properties are investigated.

In [43] and in her PhD Thesis [41] J. Lebedinska introduces the concept of a fuzzy matrix and investigates some properties of fuzzy matrices. At this point we have to note that Lebedinska's concept of a fuzzy matrix is different from other concepts of fuzzy matrices known to us and is a certain extension of Rhon's interval matrix, see [68]. In particular, she is interested in the problem of the inverse fuzzy matrix. Methods of calculation of fuzzy inverse matrices in some special cases are developed. The interest in the problem of fuzzy inverse is mainly provoked by the necessity of applications of fuzzy matrices for the study of equations with fuzzy variables [43].

Another direction of research in "fuzzy mathematics" in our group is the 
problem of approximation under imprecisely given information, that is certain kinds of fuzzy approximation problems. The principal aim of our work in this direction is to lay the basis for a fuzzy approach to extremal problem of approximation theory. We consider problems of approximation by an information in case when this information is fuzzy, and develop for this case the idea of an optimal error method of approximation (the method whose error is the minimum of the errors of all methods for a given problem) and the concept of a central algorithm (which is always an optimal error algorithm and in the crisp case is useful in practice as well as in the general theory). The first papers in this direction include $[5,6,7]$. Lately some new work was done in this area.

Recently the field of our interest included an alternative concept of fuzzy sets - so called rough sets introduced in 1983 by Z. Pawlak [58] and provoked interest both among "pure" mathematicians and "applied scientists", as well as the syntheses of the both concepts - fuzzy rough sets and rough fuzzy sets. In our work [85] the foundations of a general theory incorporating fuzzy sets, categories related to topology (both fuzzy and crisp) and rough sets (also both fuzzy and crisp) are being developed. In the same paper the introduced concepts where illustrated by many examples of different nature. The properties of rough sets their lattice-type structure and and their applications in different practical problems were studied in Msc. Theses by A. Elıkins and M. Bērziņš (2009) and presented at several conferences.

\section{Acknowledgment}

The author expresses gratitude to his colleagues Svetlana Asmuss and Olga Grigorenko for reading the preliminary version of this work carefully and making some valuable comments concerning the content and exposition of the material. The author is thankful also to the other members of our Seminar Ingrida Uljane, Sergejs Solovjovs, Julija Lebedinska, Irina Zvina, Večislavs Ruža, Pavels Orlovs, Aleksandrs Eļkins, and Svetlana Komara for many discussions related to the topics considered in this work. The author is grateful to the referee for valuable comments and in particular for his suggestion to extend the last section in order to give more information about "Fuzzy Mathematics" in Latvia. The author is also very grateful to the organizing committee of the $16^{\text {th }}$ Conference on Mathematical Modelling and Analysis and in particular to its chairman professor R. Čiegis for inviting him to give a plenary talk at the conference. The contents of this talk became the basis for the present work.

\section{References}

[1] K.P. Adlassing. Fuzzy set theory in medical diagnosis. IEEE Transactions on Systems, Man and Cybernetics SMS, 16(2):260-265, 1996. Doi:10.1109/TSMC.1986.4308946.

[2] M. Akay, M. Cohen and D. Hudson. Fuzzy sets in life sciences. Fuzzy Sets and Systems, 90(2):219-224, 1997. Doi:10.1016/S0165-0114(97)00089-4.

[3] Arbib. Review article on fuzzy set theory. Bull. Amer. Math. Soc., 83:946-951, 1977. 
[4] Aristotle. The Organon. New York, Penguin, Classics, 162.

[5] S. Asmuss and A. Šstak. Extremal problems of approximation of fuzzy sets. Acta Universitatis Latviensis. Mathematics, 606:9-18, 1997.

[6] S. Asmuss and A. Šostak. Extremal problems of approximation theory in fuzzy context. Fuzzy Sets and Systems, 105(2):249-257, 1999. Doi:10.1016/S0165-0114(98)00324-8.

[7] S. Asmuss and A. Šostak. On central algorithms of approximation under fuzzy information. Fuzzy Sets and Systems, 155(1):150-163, 2005. Doi:10.1016/j.fss.2005.05.018.

[8] R. Bellman and L.A. Zadeh. Decision making in a fuzzy environment. Management Science, 17(4):B141-B164, 1970. Doi:10.1287/mnsc.17.4.B141.

[9] G. Birkhoff. Lattice Theory. Amer. Math. Soc., Coll. Publ, Amer. Math. Soc. RI, 1973.

[10] M. Black. Vagueness. Philos. Sci., 4(4):427-455, 1937. Doi:10.1086/286476.

[11] D. Butnariu and E.P. Klement. Triangular norm based measures. In E. Pap(Ed.), Handbook on Measure Theory, pp. 947-1010. Elsevier, Amsterdam, 2002. Doi:10.1016/B978-044450263-6/50024-5.

[12] C.L. Chang. Fuzzy topological spaces. J. Math. Anal. Appl., 24:182-190, 1968.

[13] P. Demicco and C.J. Klir (Eds.). Fuzzy Logic in Geology. Elsevier Publ., 2004.

[14] D. Dubois, W. Ostasiewicz and H. Prade. Fuzzy sets: History and basic notions. In D. Dubois and H. Prade(Eds.), Fundamentals of Fuzzy Sets, pp. 947-1010. Kluwer Acad. Publ., Boston, Dodreht, 1999.

[15] D. Dubois and H. Prade. Towards fuzzy differential calculus. Part I. Integration of fuzzy mappings. Fuzzy Sets and Systems, 8(1):1-17, 1982. Doi:10.1016/0165-0114(82)90025-2.

[16] D. Dubois and H. Prade. Editorial. Fuzzy Sets and Systems, 24(3):259-262, 1987. Doi:10.1016/0165-0114(87)90026-1.

[17] R. Engelking. General Topology. PWN, Warszawa, 1977.

[18] A. Esi. On some new paranormed sequence spaces of fuzzy real numbers defined by Orlicz functions and statistical convergence. Math. Model. Anal., 11(4):379388, 2006. Doi:10.1080/13926292.2006.9637325.

[19] M. Et, Y. Altin and H. Altinok. On almost statistical convergence of generalized difference sequences fuzzy numbers. Math. Model. Anal., 10(4):345-353, 2005. Doi:10.1080/13926292.2005.9637292.

[20] T.E. Gantner, R.C. Steinlage and R.H. Warren. Compactness in fuzzy topological spaces. J. Math. Anal. Appl., 62(3):547-562, 1978. Doi:10.1016/0022-247X(78)90148-8.

[21] J. Gutierrez Garcia, M.A. de Prada Vicente and A. Šostak. A unified approach to the concept of fuzzy $L$-uniform space. In S.E. Rodabaugh and E.P. Klement(Eds.), Topological and Algebraic Structures in Fuzzy Sets: A Handbook of Recent Developments in the Mathematics of Fuzzy Sets, pp. 81-114. Kluwer Acad. Publ., Dodrecht, Boston, London, 2003.

[22] J.A. Goguen. L-fuzzy sets. J. Math. Anal. Appl., 18(1):145-174, 1967. Doi:10.1016/0022-247X(67)90189-8.

[23] J.A. Goguen. Fuzzy tychonoff theorem. J. Math. Anal. Appl., 43(3):734-742, 1973. Doi:10.1016/0022-247X(73)90288-6. 
[24] S. Gottwald. Many-valued logic and fuzzy set theory. In S.E. Rodabaugh and U.Höhle(Eds.), Mathematics of Fuzzy Sets: Logic, Topology and Measure Theory, pp. 5-90. Kluwer Acad. Publ., Boston, Dodreht, London, 1999.

[25] O. Grigorenko. Degree of monotonicity in aggregation process. In Proc. of WCCI 2010 IEEE World Congress on Computational Intelligence (WCCI 2010), pp. 1080-1087, 2010.

[26] O. Grigorenko. Involving fuzzy order in the definition of monotonicity for aggregation function. In M. Kolvačeva(Ed.), Proceedings of the APLIMAT 2011 Conference, pp. 547-556, Bratislava, 2011.

[27] U. Höhle. Fuzzy real numbers as Dedekind cuts with respect to multiple-valued logic. Fuzzy Sets and Systems, 24(3):263-278, 1987. Doi:10.1016/0165-0114(87)90027-3.

[28] U. Höhle and A. Šostak. Axiomatic foundations of fixed basis fuzzy topology. In S.E. Rodabaugh and U. Höhle(Eds.), Mathematics of Fuzzy Sets: Logic, Topology and Measure Theory, pp. 81-114. Kluwer Acad. Publ., Boston, Dodreht, London, 1999.

[29] B. Hutton. Normality in fuzzy topological spaces. J. Math. Anal. Appl., 50(1):74-79, 1975. Doi:10.1016/0022-247X(75)90039-6.

[30] B. Hutton. Products of fuzzy topological spaces. Topology and Appl., 11(1):5967, 1980. Doi:10.1016/0166-8641(80)90017-6.

[31] O. Kaleva. Fuzzy differential equations. Fuzzy Sets and Systems, 24(3):301-317, 1987. Doi:10.1016/0165-0114(87)90029-7.

[32] O. Kaleva. The cauchy problem for fuzzy differential equations. Fuzzy Sets and Systems, 35(3):389-396, 1990. Doi:10.1016/0165-0114(90)90010-4.

[33] J.L. Kelly. General Topology. D. Van Nostrand Company, Toronto, New York, London, 1957.

[34] S. Kleene. Introduction to Metamathematics. Elsevier Publ., 1952.

[35] E.P. Klement. Fuzzy measures assuming their values in the set of fuzzy numbers. J. Math. Anal. Appl., 93(2):312-323, 1983. Doi:10.1016/0022-247X(83)90176-2.

[36] E.P. Klement, R. Mesiar and E. Pap. Triangular Norms. Kluwer Acad. Publ., Dordrecht, Boston, London, 2000.

[37] E.P. Klement, W. Schwyhla and R. Lowen. Fuzzy probability measures. Fuzzy Sets and Systems, 5(2):21-30, 1981. Doi:10.1016/0165-0114(81)90031-2.

[38] E.P. Klement and S. Weber. Generalized measures. Fuzzy Sets and Systems, 40(2):375-394, 1991. Doi:10.1016/0165-0114(91)90166-N.

[39] B. Kosko. Fuzzy Thinking. Hyperion, New York, 1993.

[40] T. Kubiak. On fuzzy topologies. PhD thesis, Adam Mickiewicz University, Poznan, Poland, 1985.

[41] J. Lebedinska. Fuzzy matrices and generalized aggregation operators: theoretical foundations and possible applications. $\mathrm{PhD}$ thesis, University of Latvia, Riga, 2010.

[42] J. Lebedinska. $\gamma$-aggregation operators and some aspects of generalized aggregation problem. Math. Model. Anal., 15(1):83-96, 2010. Doi:10.3846/1392-6292.2010.15.83-96. 
[43] J. Lebedinska. On another view of an inverse of an interval matrix. Soft Computing - A Fusion of Foundations, Methodologies and Applications, 14(10):10431046, 2010.

[44] J. Lebedinska. T-extension as a method of construction of a generalized aggregation operator. Kybernetika, 46(6):1078-1097, 2010.

[45] X. Liu and G. Zhang. Fuzzy-valued fuzzy measure and latticevalued fuzzy integral. Fuzzy Sets and Systems, 62(3):319-332, 1994. Doi:10.1016/0165-0114(94)90116-3.

[46] R. Lowen. Fuzzy topological spaces and fuzzy compactness. J. Math. Anal. Appl., 56:153-176, 1976.

[47] J. Łukasiewicz. Zur geschichte der aussagenlogik. Erkentnis, 5(1):111-135, 1935. Doi:10.1007/BF00172288.

[48] S. Markin and A. Šostak. Another approach to the concept of a fuzzy proximity. Suppl. Rend. Circ. Matem. Palermo, Ser. II, 29:530-551, 1992.

[49] K. Menger. Probabilistic geometry. In Proc. N.A.S., volume 37, pp. 226-229, 1951.

[50] M. Mizumoto and K. Tanaka. The four operations of arithmetics on fuzzy numbers. Systems-Comput.-Controls, 7:73-81, 1976.

[51] J.N. Mordenson and D.S. Malik. Fuzzy Commutative Algebra. World Publ., 1998.

[52] M. Mursaleen and M. Başanr. On some new sequence spaces of fuzzy numbers. Indian J. Pure Appl. Math., 34(9):1351-1357, 2003.

[53] C.V. Negoita and D.A. Ralescu. Application of fuzzy sets to system analysis. Interdisciplinary Systems Research Papers, 11, New York, Budapest, Basel, Schtutgart, Halsed Press, 1975.

[54] H.T. Nguen and E. Walker. A First Course in Fuzzy Logic. CRC press, New York, London, Tokyo, second edition, 1999.

[55] V. Novak and I. Perfilieva. Discovering the World of Fuzzy Logic. Kluwer Acad. Publ., Dodrecht, 2000.

[56] V. Novak and I. Perfilieva. Discovering the world of fuzzy logic. Stud. Fuzziness Soft Comput., 57, Springer Verlag, Heidelberg, 2000.

[57] P. Orlovs. On aggregation of $L$-fuzzy real numbers. In M. Kolvačeva(Ed.), Proceedings of the APLIMAT 2011 Conference, pp. 595-604, Bratislava, 2011.

[58] Z. Pawlak. Rough sets. Int. J. of Computers and Information Sci., 11(5):341356, 1982. Doi:10.1007/BF01001956.

[59] W. Pedrycz. Fuzzy Modelling: Paradigms and Practices. Kluwer Acad. Publ., Dordrecht, 1996.

[60] C.S. Peirce. Collected Papers. Harvard University Press, Cambridge, 1932.

[61] E.L. Post. Introduction to a general theory of elementary propositions. Amer. J. Math., 43(3):165-185, 1921. Doi:10.2307/2370324.

[62] P.-M. Pu and Y.-M. Liu. Fuzzy topology. Part I. Neighborhood structure of a fuzzy point and Moore-Smith convergence. J. Math. Anal. Appl., 76(2):571599, 1980. Doi:10.1016/0022-247X(80)90048-7.

[63] M.L. Puri and D.A. Ralescu. Differentials of fuzzy functions. J. Math. Anal. Appl., 91(2):552-558, 1983. Doi:10.1016/0022-247X(83)90169-5. 
[64] D. Ralescu and G. Adams. The fuzzy integrals. J. Math. Anal. Appl., 75(2):562570, 1980. Doi:10.1016/0022-247X(80)90101-8.

[65] S.E. Rodabaugh. The categorical accommodation of various notions of fuzzy topology. Fuzzy Sets and Systems, 9(1-3):163-183, 1983. Doi:10.1016/S0165-0114(83)80026-8.

[66] S.E. Rodabaugh. Complete fuzzy topological hyperfields and fuzzy multiplication in the fuzzy real lines. Fuzzy Sets and Systems, 15(3):285-310, 1985. Doi:10.1016/0165-0114(85)90022-3.

[67] S.E. Rodabaugh. Variable-basis fuzzy topology. In S.E. Rodabaugh and U. Höhle(Eds.), Mathematics of Fuzzy Sets: Logic, Topology and Measure Theory, pp. 481-552. Kluwer Academic Publ., Boston, Dodreht, London, 1999.

[68] J. Rohn. Regularity of interval matrices and theorems of the alternatives. Reliab. Comput., 12(2):99-105, 2005. Doi:10.1007/s11155-006-4877-z.

[69] A. Rosenfeld. Fuzzy groups. J. Math. Anal. Appl., 35(3):512-517, 1970. Doi:10.1016/0022-247X(71)90199-5.

[70] B. Russel. Introduction to Mathematical Philosophy. Simon \& Schuster, New York, 1917.

[71] V. Ruza. On an $L$-fuzzy valued integral with respect to an $L$-fuzzy valued $T$ measure. In M. Kolvačeva(Ed.), Proceedings of the APLIMAT 2011 Conference, pp. 605-614, Bratislava, 2011.

[72] V. Ruzha and S. Asmuss. A construction of a fuzzy valued measure based on minimum $t$-norm. In Štepnička, V. Novak and U. Bodenhofer(Eds.), Proceedings of the 5th EUSFLAT Conference, pp. 175-178, Ostrava, 2007.

[73] V. Ruzha and S. Asmuss. A construction of an $L$-fuzzy valued measure of $L$-fuzzy sets. In Proceedings of the 5th EUSFLAT Conference, pp. 1735-1739, Lisbon, 2009.

[74] S. Seikalla. The initial fuzzy value problem. Fuzzy Sets and Systems, 24:389396, 1987.

[75] S. Solovjov. On a generalization of Goguen's category SET(L). Fuzzy Sets and Systems, 158(4):367-385, 2007. Doi:10.1016/j.fss.2006.09.007.

[76] S. Solovjov. Sobriety and spatiality in varieties of algebras. Fuzzy Sets and Systems, 159(19):2567-2585, 2008. Doi:10.1016/j.fss.2008.02.010.

[77] S. Solovjov. On ordered categories as framework for fuzzification of algebraic and topological structures. Fuzzy Sets and Systems, 160(20):2910-2925, 2009. Doi:10.1016/j.fss.2009.02.009.

[78] S. Solovjov. From quantale algebras to topological spaces: Fixed and variable based approach. Fuzzy Sets and Systems, 161(9):1270-1287, 2010. Doi:10.1016/j.fss.2010.01.004.

[79] S. Solovjovs. On a Categorical Generalization of the Concept of Fuzzy Set. PhD thesis, University of Bremen, Bremen, 2007.

[80] Šostak. On some fuzzy categories related to category L-TOP of $L$-topological spaces. In S.E. Rodabaugh and E.P. Klement(Eds.), Topological and Algebraic Structures in Fuzzy Sets: A Handbook of Recent Developments in the Mathematics of Fuzzy Sets, pp. 309-336. Kluwer Acad. Publ., Dodrecht, Boston, London, 2003. 
[81] Šostak. $L$-valued categories: Generalities and examples related to algebra and topology. In W. Gáhler and G. Preuss(Eds.), Categorical Structures and Their Applications, pp. 291-313. World Scientific, New Jersey, London, Singapore, 2004.

[82] A. Šostak. On a fuzzy topological structure. Suppl. Rend. Circ. Matem. Palermo, 11:89-103, 1985.

[83] A. Šstak. Two decades of fuzzy topology: basic ideas, notions and results. Russian Math. Surveys, 44:125-186, 1989. Doi:10.1070/RM1989v044n06ABEH002295.

[84] A. Šostak. Basic structures of fuzzy topology. J. Math. Sci., 78(6):662-701, 1996. Doi:10.1007/BF02363065.

[85] A. Šstak. Towards the theory of $\mathbb{M}$-approximate systems: Fundamentals and examples. Fuzzy Sets and Systems, 161(18):2440-2461, 2010. Doi:10.1016/j.fss.2010.05.010.

[86] M. Sugeno. Theory of fuzzy integrals and its application. PhD thesis, Tokyo Institute of Technology, Tokyo, Japan, 1974.

[87] B.C. Tripathy and S. Borgohain. Difference sequence space $m\left(M, p h i, \Delta_{m}^{n}, p\right)^{F}$ of fuzzy real numbers. Math. Model. Anal., 13(4):577-586, 2008. Doi:10.3846/1392-6292.2008.13.577-586.

[88] I. Uljane. On some categories of L-valued sets and many-valued topologies: theoretical foundations. PhD thesis, University of Latvia, Riga, Latvia, 2008.

[89] I. Uljane. On some fuzzy categories of many-valued topological spaces. In Proceedings of IFSA-EUSFLAT, pp. 921-924, 2009.

[90] I. Uljane and A.P. Šstaks. On a category on L-valued equalities on L-sets. Journal of Electrical Engineering, 55:60-64, 2004.

[91] I. Uljane. On the order type $L$-valued relations on $L$-powersets. Mathware and Soft Computing, 1(3):183-199, 2007.

[92] H.-C. Wu. Fuzzy-valued integrals of fuzzy-valued measurable functions with respect to fuzzy-valued measures based on closed intervals. Fuzzy Sets and Systems, 87(1):65-78, 1997. Doi:10.1016/0165-0114(95)00409-2.

[93] F. Zadeh. My Life and Travels with the Father of Fuzzy Logic. TSI Press, Albuquerque, NM, 1998.

[94] L.A. Zadeh. Fuzzy sets. Information and Control, 8(3):338-353, 1965. Doi:10.1016/S0019-9958(65)90241-X.

[95] L.A. Zadeh. Probability measures of fuzzy events. J. Math. Anal. Appl., 23(2):421-427, 1968. Doi:10.1016/0022-247X(68)90078-4.

[96] L.A. Zadeh. Fuzzy sets as a basis for a theory of possibility. Fuzzy Sets and Systems, 1(1):3-28, 1978. Doi:10.1016/0165-0114(78)90029-5.

[97] I. Zvina. Complete infinitely distributive lattices as topologies modulo an ideal. Acta Univ. Latviensis, 688:121-128, 2005.

[98] I. Zvina. On i-topological spaces: ganeralization of the concept of a topological space via ideals. Appl. Gen. Topol., 7(1):53-66, 2006.

[99] I. Zvina. Introduction to generalized topological spaces. Appl. Gen. Topol., 2010. Accepted

[100] I. Zvina. Topologies modulo compatible ideal: set-theoretical study and representation in locale theory. PhD thesis, University of Latvia, Riga, Latvia, 2010. 